\title{
MULTIPLICATION OPERATORS AND DYNAMICAL SYSTEMS ON WEIGHTED SPACES OF CROSS-SECTIONS
}

\author{
R. K. SINGH AND J. S. MANHAS
}

(Communicated by Palle E. T. Jorgensen)

\begin{abstract}
Let $Y$ be a Hausdorff topological space, let $V$ be a system of weights on $Y$, and let $L V_{0}(Y)$ and $L V_{b}(Y)$ be the weighted locally convex spaces of cross-sections with a topology generated by seminorms which are weighted analogues of the supremum norm. In the present paper, we characterise the multiplication operators on the spaces $L V_{0}(Y)$ and $L V_{\mathrm{b}}(Y)$ induced by the scalar-valued, the vector-valued, and the operator-valued mappings. A (linear) dynamical system on the weighted spaces of cross-sections is obtained as an application of the theory of the multiplication operators. Many examples are given to illustrate the theory.
\end{abstract}

\section{INTRODUCTION}

Let $Y$ be a nonempty set, and let $\left(Y,\left(F_{y}\right)_{y \in Y}\right)$ be a vector-fibration over $Y$, where each $F_{y}$ is an algebra over the field $\mathbb{K}$ (where $\mathbb{K}=\mathbb{R}$ or $\mathbb{C}$ ). We denote by $\left(T\left(F_{y}\right)\right)_{y \in Y}$ a family of vector spaces of linear transformations from $F_{y}$ to itself. The Cartesian product $\prod_{y \in Y} F_{y}$ of the family $\left(F_{y}\right)_{y \in Y}$ is a vector space under pointwise operations. Any element of the Cartesian product $\prod_{y \in Y} F_{y}$ is known as a cross-section over $Y$. Let $L(Y)$ be a topological vector space of cross-sections over $Y$. Let $\pi$ be a complex-valued function on $Y$, $\psi$ be a cross-section over $Y$, and $\eta: Y \rightarrow \bigcup_{y \in Y}\left(T\left(F_{y}\right)\right)$ be a mapping such that $\eta(y) \in T\left(F_{y}\right)$ for each $y \in Y$. Then the scalar multiplication, the vector multiplication, and the composition of mappings give rise to three linear transformations $M_{\pi}, M_{\psi}$, and $M_{\eta}$ from $L(Y)$ to the linear space $\prod_{y \in Y} F_{y}$ of all cross-sections over $Y$, defined as $M_{\pi} f=\pi f, M_{\psi} f=\psi f$, and $M_{\eta} f=\eta f$, where the product of functions is defined pointwise. In case $M_{\pi}, M_{\psi}$, and $M_{\eta}$ take $L(Y)$ into itself and they are continuous, they are called the multiplication operators on $L(Y)$ induced by the mappings $\pi, \psi$, and $\eta$, respectively. These operators have been the subject of study for a long time on different function spaces, especially on $L^{p}$-spaces and $H^{p}$-spaces, and they have played a very important role in the study of operators on Hilbert spaces. For more details about these operators we refer to Abrahamse [1].

Received by the editors February 24, 1992.

1991 Mathematics Subject Classification. Primary 47B38; Secondary 46E40, 34C35.

Key words and phrases. Weights, cross-sections, seminorms, vector-valued and operator-valued mappings, multiplication operators, dynamical systems. 
We initiated the study of these operators on the weighted function spaces in [7]. This paper is a continuation of our earlier papers [8, 9] in which we studied the multiplication operators on the weighted spaces of vector-valued functions. In this note we endeavour to study the multiplication operators on the weighted spaces of cross-sections induced by the scalar-valued, vector-valued, and operator-valued mappings. The study of these operators on these function spaces comes in contact with topological dynamics. Section 2 of this paper contains preliminaries, and in the third section we characterise the functions which induce the multiplication operators on the weighted spaces of cross-sections. We illustrate the theory in $\S 4$ by giving examples of the functions which induce or do not induce the multiplication operators on the weighted locally convex spaces of cross-sections. Finally, we end this paper with $\S 5$ by obtaining a (linear) dynamical system on the weighted function spaces of cross-sections.

\section{Preliminaries}

Let $Y$ be a Hausdorff topological space. A vector-fibration over $Y$ is a pair $\left(Y,\left(F_{y}\right)_{y \in Y}\right)$, where each $F_{y}$ is a vector space over the field $\mathbb{K}$ (where $\mathbb{K}=\mathbb{R}$ or $\mathbb{C})$. A cross-section over $Y$ is then any element of the Cartesian product $\prod_{y \in Y} F_{y}$. The Cartesian product $\prod_{y \in Y} F_{y}$ is made a vector space in the usual way, and a vector space of cross-sections over $Y$ is, by definition, any vector subspace of $\prod_{y \in Y} F_{y}$.

By a 'weight' on $Y$ we mean a function $v$ on $Y$ such that $v(y)$ is a seminorm on $F_{y}$ for each $y \in Y$. For our convenience, we shall use the notation $v_{y}$ for the seminorm $v(y)$ for each $y \in Y$. By $v \leq u$ we mean that $v_{y} \leq u_{y}$ for every $y \in Y$. A set $V$ of weights on $Y$ is said to be directed if, for every pair $u, v \in V$ and $\lambda>0$, there exists $w \in V$ such that $\lambda u \leq w$ and $\lambda v \leq w$. Hereafter we assume that each set of weights is directed. We write $V>0$, if given $y \in Y$ and $x \in F_{y}$, there is some $v \in V$ for which $v_{y}(x)>0$. A set $V$ of weights on $Y$ which additionally satisfies $V>0$ will be referred to as a system of weights on $Y$. If $f$ is a cross-section over $Y$ and $v$ is a weight on $Y$, then we will denote by $v[f]$ the positive-valued function on $Y$ which takes $y$ into $v_{y}[f(y)]$. We denote by $L(Y)$ a vector space of cross-sections over $Y$. Now the weighted spaces of cross-sections over $Y$ with respect to the system of weights $V$ are introduced as follows:

$$
\begin{array}{r}
L V_{0}(Y)=\{f \in L(Y): v[f] \text { is upper semicontinuous and vanishes } \\
\text { at infinity on } Y \text { for each } v \in V\}
\end{array}
$$

and

$$
L V_{\mathrm{b}}(Y)=\{f \in L(Y): v[f] \text { is a bounded function on } Y \text { for each } v \in V\} \text {. }
$$

Obviously, $L V_{0}(Y)$ and $L V_{\mathrm{b}}(Y)$ are vector spaces and $L V_{0}(Y) \subseteq L V_{\mathrm{b}}(Y)$. Now, for $v \in V$ and $f \in L(Y)$, if we put $\|f\|_{v}=\sup \left\{v_{y}[f(y)]: y \in Y\right\}$, then $\|\cdot\|_{v}$ can be regarded as a seminorm on either $L V_{\mathrm{b}}(Y)$ or $L V_{0}(Y)$, and the family of seminorms $\left\{\|\cdot\|_{v}: v \in V\right\}$ defines a Hausdorff locally convex topology on each of these spaces. This topology is denoted by $\tau_{V}$, and the vector spaces endowed with $\tau_{V}$ are called the weighted locally convex spaces of cross-sections. Since $V$ is a directed set of weights, $\tau_{V}$ has a basis of closed absolutely convex neighborhoods of the origin of the form

$$
B_{v}=\left\{f \in L V_{\mathrm{b}}(Y):\|f\|_{v} \leq 1\right\} .
$$


For illustrations and details about these weighted spaces of cross-sections we refer to [2-6].

\section{FUNCTIONS INDUCING THE MULTIPLICATION OPERATORS ON THE WEIGHTED SPACES OF CROSS-SECTIONS}

In this section we characterise the scalar-valued, vector-valued, and operatorvalued mappings which induce the multiplication operators on $L V_{0}(Y)$ and $L V_{\mathrm{b}}(Y)$. We shall generalise some of the results obtained in $[8,9]$ to the weighted spaces of cross-sections.

In the following theorem we characterise the scalar-valued mappings $\pi: Y \rightarrow$ $\mathbb{C}$ which induce the multiplication operators on $L V_{0}(Y)$.

Theorem 3.1. Let $\pi: Y \rightarrow \mathbb{C}$ be a mapping. Then $M_{\pi}: L V_{0}(Y) \rightarrow L V_{0}(Y)$ is a multiplication operator if and only if for every $v \in V$ there exists $u \in V$ such that

$$
|\pi(y)| v_{y}(x) \leq u_{y}(x) \text { for every } y \in Y \text { and } x \in F_{y} .
$$

Proof. First of all, suppose that for every $v \in V$ there exists $u \in V$ such that

$$
|\pi(y)| v_{y}(x) \leq u_{y}(x) \text { for every } y \in Y \text { and } x \in F_{y} .
$$

We shall show that $M_{\pi}: L V_{0}(Y) \rightarrow L V_{0}(Y)$ is a continuous linear operator. Clearly, $M_{\pi}$ is a linear operator on $L V_{0}(Y)$. To show that $M_{\pi}$ is a continuous operator on $L V_{0}(Y)$, it is enough to show that $M_{\pi}$ is continuous at the origin. Let $\left\{f_{\alpha}\right\}$ be a net in $L V_{0}(Y)$ such that $\left\|f_{\alpha}\right\|_{v} \rightarrow 0$ for every $v \in V$. Then

$$
\begin{aligned}
\left\|\pi f_{\alpha}\right\|_{v} & =\sup \left\{v_{y}\left[\pi(y) f_{\alpha}(y)\right]: y \in Y\right\} \\
& \leq \sup \left\{u_{y}\left[f_{\alpha}(y)\right]: y \in Y\right\}=\left\|f_{\alpha}\right\|_{u} \rightarrow 0 .
\end{aligned}
$$

This proves that $M_{\pi}$ is continuous at the origin, and hence $M_{\pi}$ is a continuous linear operator on $L V_{0}(Y)$.

Conversely, if $M_{\pi}: L V_{0}(Y) \rightarrow L V_{0}(Y)$ is a multiplication operator, then for $v \in V$ there exists $u \in V$ such that $M_{\pi}\left(B_{u}\right) \subseteq B_{v}$. We claim that

$$
|\pi(y)| v_{y}(x) \leq u_{y}(x) \text { for every } y \in Y \text { and } x \in F_{y} \text {. }
$$

Fix $y_{0} \in Y$ and $x_{0} \in F_{y_{0}}$. Set $u_{y_{0}}\left(x_{0}\right)=\varepsilon$. In case $\varepsilon>0$, we define the function $g: Y \rightarrow \bigcup_{y \in Y} F_{y}$ as

$$
g(y)= \begin{cases}\varepsilon^{-1} x_{0}, & y=y_{0}, \\ 0, & y \neq y_{0} .\end{cases}
$$

Clearly, $g$ is a cross-section and $g \in L V_{0}(Y)$. Also, $g \in B_{u}$ and $\pi g \in B_{v}$. From this it follows that $v_{y}[\pi(y) g(y)] \leq 1$ for every $y \in Y$. Further, it implies that $|\pi(y)| v_{y}[g(y)] \leq 1$ for every $y \in Y$. Thus $\left|\pi\left(y_{0}\right)\right| v_{y_{0}}\left(x_{0}\right) \leq u_{y_{0}}\left(x_{0}\right)$. Since $y_{0}$ and $x_{0}$ are arbitrary, in this case our claim is established. In case $u_{y_{0}}\left(x_{0}\right)=$ 0 , we have to prove that $\left|\pi\left(y_{0}\right)\right| v_{y_{0}}\left(x_{0}\right)=0$. Suppose that $\left|\pi\left(y_{0}\right)\right| v_{y_{0}}\left(x_{0}\right)>0$, and put

$$
\varepsilon=\frac{\left|\pi\left(y_{0}\right)\right| v_{y_{0}}\left(x_{0}\right)}{2} .
$$

Define the function $g: Y \rightarrow \bigcup_{y \in Y} F_{y}$ as

$$
g(y)= \begin{cases}\varepsilon^{-1} x_{0}, & y=y_{0}, \\ 0, & y \neq y_{0} .\end{cases}
$$


Then $g \in B_{u}$, and hence $\pi g \in B_{v}$. From this it follows that

$$
|\pi(y)| v_{y}[g(y)] \leq 1 \quad \text { for every } y \in Y .
$$

Hence

$$
\left|\pi\left(y_{0}\right)\right| v_{y_{0}}\left(x_{0}\right) \leq \frac{\left|\pi\left(y_{0}\right)\right| v_{y_{0}}\left(x_{0}\right)}{2},
$$

which is impossible, and hence in this case, too, our claim is established. This completes the proof of the theorem.

Corollary 3.2. If $\pi: Y \rightarrow \mathbb{C}$ is a bounded mapping, then $M_{\pi}: L V_{0}(Y) \rightarrow L V_{0}(Y)$ is a multiplication operator.

Proof. The proof follows from Theorem 3.1.

In the next theorem we characterise the vector-valued mappings which induce the multiplication operators on $L V_{0}(Y)$. In the following theorem we shall consider the vector-fibration as a pair $\left(Y,\left(F_{y}\right)_{y \in Y}\right)$, where $\left(F_{y}\right)_{y \in Y}$ is a family of (topological) algebras over the same field $\mathbb{K}$.

Theorem 3.3. Let $\pi: Y \rightarrow \bigcup_{y \in Y} F_{y}$ be a mapping such that $\pi(y) \in F_{y}$ for every $y \in Y$. Then $M_{\pi}: L V_{0}(Y) \rightarrow L V_{0}(Y)$ is a multiplication operator if and only if for every $v \in V$ there exists $u \in V$ such that

$$
v_{y}(\pi(y) x) \leq u_{y}(x) \text { for every } y \in Y \text { and } x \in F_{y} .
$$

Proof. Suppose the condition of the theorem holds, and suppose $\left\{f_{\alpha}\right\}$ is a net in $L V_{0}(Y)$ such that $\left\|f_{\alpha}\right\|_{v} \rightarrow 0$ for every $v \in V$. Then

$$
\begin{aligned}
\left\|\pi f_{\alpha}\right\|_{v} & =\sup \left\{v_{y}\left[\pi(y) f_{\alpha}(y)\right]: y \in Y\right\} \\
& \leq \sup \left\{u_{y}\left[f_{\alpha}(y)\right]: y \in Y\right\}=\left\|f_{\alpha}\right\|_{u} \rightarrow 0 .
\end{aligned}
$$

This proves the continuity of $M_{\pi}$ at the origin and hence on $L V_{0}(Y)$.

Conversely, suppose $M_{\pi}$ is a multiplication operator on $L V_{0}(Y)$. We need to show that for every $v \in V$ there exists $u \in V$ such that $v_{y}(\pi(y) x) \leq u_{y}(x)$ for every $y \in Y$ and $x \in F_{y}$. This inequality can be established in the same way as we did in the converse part of Theorem 3.1.

In the next theorem we characterise the operator-valued mappings inducing multiplication operators on $L V_{0}(Y)$.

Theorem 3.4. Let $\pi: Y \rightarrow \bigcup_{y \in Y}\left(T\left(F_{y}\right)\right)$ be a mapping such that $\pi_{y}(=\pi(y))$ belongs to $T\left(F_{y}\right)$ for every $y \in Y$. Then $M_{\pi}: L V_{0}(y) \rightarrow L V_{0}(Y)$ is a multiplication operator if and only if for every $v \in V$ there exists $u \in V$ such that

$$
v_{y}\left(\pi_{y}(x)\right) \leq u_{y}(x) \text { for every } y \in Y \text { and } x \in F_{y} .
$$

Proof. Suppose that the condition of the theorem holds. To show that $M_{\pi}$ : $L V_{0}(Y) \rightarrow L V_{0}(Y)$ is a multiplication operator, it is enough to show that $M_{\pi}$ is continuous at the origin. To show this, let $\left\{f_{\alpha}\right\}$ be a net in $L V_{0}(Y)$ such that $\left\|f_{\alpha}\right\|_{v} \rightarrow 0$ for every $v \in V$. Then

$$
\begin{aligned}
\left\|\pi f_{\alpha}\right\|_{v} & =\sup \left\{v_{y}\left[\pi_{y}\left(f_{\alpha}(y)\right)\right]: y \in Y\right\} \\
& \leq \sup \left\{u_{y}\left[f_{\alpha}(y)\right]: y \in Y\right\}=\left\|f_{\alpha}\right\|_{u} \rightarrow 0 .
\end{aligned}
$$

This proves the continuity of $M_{\pi}$ at the origin and hence on $L V_{0}(Y)$. 
Conversely, if $M_{\pi}$ is a multiplication operator on $L V_{0}(Y)$, then we can easily establish that for every $v \in V$ there exists $u \in V$ such that

$$
v_{y}\left(\pi_{y}(x)\right) \leq u_{y}(x) \text { for every } y \in Y \text { and } x \in F_{y} .
$$

With this the proof of the theorem is completed.

Remark 3.5. If we replace the weighted space $L V_{0}(Y)$ of cross-sections by the weighted space $L V_{\mathrm{b}}(Y)$ of cross-sections, even then all the results obtained so far go through.

Now we shall give some examples of the mappings which induce or do not induce the multiplication operators on the weighted space $L V_{\mathrm{b}}(Y)$ of crosssections in the following section.

\section{EXAMPLES}

Example 4.1. Let $\pi: \mathbb{N} \rightarrow \mathbb{C}$ be the mapping defined as $\pi(n)=1 / n$ for every $n \in \mathbb{N}$. Let $\left(\mathbb{N},\left(F_{n}\right)_{n \in \mathbb{N}}\right)$ be the vector-fibration over $\mathbb{N}$, where, for every $n \in \mathbb{N}, F_{n}=\mathbb{C}^{n}$ with the usual norm. Now we define the function $v$ on $\mathbb{N}$ as $v_{n}=\|\cdot\|_{n}$. Then clearly $v$ is a weight on $\mathbb{N}$, and the set $V=\{\lambda v: \lambda \geq 0\}$ is a system of weights on $\mathbb{N}$. Since the mapping $\pi$ is bounded, in view of Corollary 3.2 we conclude that $M_{\pi}$ is a multiplication operator on $L V_{\mathrm{b}}(\mathbb{N})$.

On the other hand, if we define $\pi: \mathbb{N} \rightarrow \mathbb{C}$ as $\pi(n)=n^{2}$ for every $n \in \mathbb{N}$, then $M_{\pi}$ is not a multiplication operator on $L V_{\mathrm{b}}(\mathbb{N})$. In this case $M_{\pi}$ is not even an into map. To see this, we define the function $g: \mathbb{N} \rightarrow \bigcup_{n \in \mathbb{N}} \mathbb{C}^{n}$ as $g(n)=\left(0,0, \ldots, \frac{1}{n}\right) \in \mathbb{C}^{n}$. Then clearly $g \in L V_{\mathrm{b}}(\mathbb{N})$, but $\pi g \notin L V_{\mathrm{b}}(\mathbb{N})$ because $v[\pi \cdot g]$ is an unbounded function on $\mathbb{N}$.

Example 4.2. For each $n \in \mathbb{N}$ let $C_{\mathrm{b}}\left(\mathbb{R}^{n}\right)$ be the normed linear space of all bounded continuous complex-valued functions on $\mathbb{R}^{n}$ with the supnorm defined as $\|f\|_{n}=\sup \left\{|f(t)|: t \in \mathbb{R}^{n}\right\}$. Consider the vector-fibration $\left(\mathbb{N},\left(C_{\mathrm{b}}\left(\mathbb{R}^{n}\right)\right)_{n \in \mathbb{N}}\right)$. For each $n \in \mathbb{N}$, let $\phi^{n}: \mathbb{R}^{n} \rightarrow \mathbb{R}^{n}$ be a continuous mapping. It can easily be seen that each $\phi^{n}$ induces the composition operator $C_{\phi^{n}}: C_{\mathrm{b}}\left(\mathbb{R}^{n}\right) \rightarrow C_{\mathrm{b}}\left(\mathbb{R}^{n}\right)$, defined as $C_{\phi^{n}} f=f \circ \phi^{n}$ for every $f \in C_{\mathrm{b}}\left(\mathbb{R}^{n}\right)$. Let $\left\{T\left(C_{\mathrm{b}}\left(\mathbb{R}^{n}\right)\right)\right\}_{n \in \mathbb{N}}$ be the family of vector spaces of bounded linear operators on $C_{\mathrm{b}}\left(\mathbb{R}^{n}\right)$. For each $n \in \mathbb{N}$ we define the function $v$ on $\mathbb{N}$ such that $v_{n}=\|\cdot\|_{n}$, where $\|\cdot\|_{n}$ is the supnorm on $C_{\mathrm{b}}\left(\mathbb{R}^{n}\right)$. Then $v$ is a weight on $\mathbb{N}$, and the set $V=\{\lambda v: \lambda \geq 0\}$ is a system of weights on $\mathbb{N}$. Now we define the mapping $\pi: \mathbb{N} \rightarrow \bigcup_{n \in \mathbb{N}}\left\{T\left(C_{\mathrm{b}}\left(\mathbb{R}^{n}\right)\right)\right\}$ as $\pi(n)=C_{\phi^{n}}$ for every $n \in \mathbb{N}$. Since $v_{n}\left(\pi_{n}(f)\right) \leq v_{n}(f)$ for every $n \in \mathbb{N}$ and $f \in C_{\mathrm{b}}\left(\mathbb{R}^{n}\right)$, in light of Theorem 3.4 we conclude that $M_{\pi}$ is a multiplication operator on $L V_{\mathrm{b}}(\mathbb{N})$.

Now we give an example of an operator-valued mapping which does not induce a multiplication operator on $L V_{\mathrm{b}}(\mathbb{N})$. For each $n \in \mathbb{N}$ we define the function $\psi^{n}: \mathbb{R}^{n} \rightarrow \mathbb{C}$ as $\psi^{n}(t)=n$ for every $t \in \mathbb{R}^{n}$. Each $\psi^{n}$ induces the multiplication operator $M_{\psi^{n}}$ on $C_{\mathrm{b}}\left(\mathbb{R}^{n}\right)$. Now we define the mapping $\pi: \mathbb{N} \rightarrow \bigcup_{n \in \mathbb{N}}\left\{T\left(C_{\mathrm{b}}\left(\mathbb{R}^{n}\right)\right)\right\}$ as $\pi(n)=M_{\psi^{n}}$ for every $n \in \mathbb{N}$. One can easily check that $M_{\pi}$ is not a multiplication operator on $L V_{\mathrm{b}}(\mathbb{N})$. In fact, $M_{\pi}$ is not even an into map. If we define the function $F: \mathbb{N} \rightarrow \bigcup_{n \in \mathbb{N}} C_{\mathrm{b}}\left(\mathbb{R}^{n}\right)$ as $F(n)=1_{n}$ for every $n \in \mathbb{N}$, where $1_{n}$ is the constant function defined by $1_{n}(t)=1$ for every $t \in \mathbb{R}^{n}$, then $F \in L V_{\mathrm{b}}(\mathbb{N})$; but $\pi F \notin L V_{\mathrm{b}}(\mathbb{N})$, since $v[\pi \cdot F]$ is unbounded. 
Example 4.3. Consider the vector-fibration $\left(\mathbb{N},\left(F_{n}\right)_{n \in \mathbb{N}}\right)$, where $F_{n}=C_{\mathrm{b}}\left(\mathbb{R}^{n}\right)$ for every $n \in \mathbb{N}$. Define the vector-valued function $\pi: \mathbb{N} \rightarrow \bigcup_{n \in \mathbb{N}} C_{\mathrm{b}}\left(\mathbb{R}^{n}\right)$ as $\pi(n)=1_{1 / n}$ for every $n \in \mathbb{N}$, where $1_{1 / n}$ is the constant function defined on $\mathbb{R}^{n}$ by $1_{1 / n}(t)=\frac{1}{n}$ for every $t \in \mathbb{R}^{n}$. Then by Theorem $3.3 M_{\pi}$ is a multiplication operator on $L V_{\mathrm{b}}(\mathbb{N})$. In case $\pi(n)(t)=n$ for every $t \in \mathbb{R}^{n}$, the function $\pi$ does not induce a multiplication operator on $L V_{b}(\mathbb{N})$. The linear transformation $M_{\pi}$ does not take $L V_{\mathrm{b}}(\mathbb{N})$ into itself. If we take the cross-section $F$ defined as $F(n)=1_{n}$ for every $n \in \mathbb{N}$, then $F \in L V_{\mathrm{b}}(\mathbb{N})$ and $\pi \cdot F \notin L V_{\mathrm{b}}(\mathbb{N})$ since $v[\pi \cdot F]$ is an unbounded function.

\section{DYNAMICAL SYSTEMS INDUCED BY THE MULTIPLICATION OPERATORS}

Throughout this section we take $Y$ to be the real line $\mathbb{R}$ (with the usual topology). Consider the vector-fibration $\left(\mathbb{R},\left(F_{t}\right)_{t \in \mathbb{R}}\right)$, where each $F_{t}$ is a Banach space over the field $\mathbb{C}$ with the norm denoted by $\|\cdot\|_{t}$. By $B\left(F_{t}\right)$ we denote the Banach algebra of all bounded linear operators on $F_{t}$ with the operator norm denoted as $\|\cdot \cdot\| \|_{t}$. Let $F_{b}(\mathbb{R})$ be the normed linear space of all bounded functions on $\mathbb{R}$ with supnorm. Fix $g \in F_{\mathrm{b}}(\mathbb{R})$, and for each $t \in \mathbb{R}$ define the linear transformation $A_{t}: F_{t} \rightarrow F_{t}$ as

$$
A_{t}(y)=g(t) y \quad \text { for every } y \in F_{t} .
$$

Then

$$
\left\|A_{t}(y)\right\|_{t}=\|g(t) y\|_{t}=|g(t)|\|y\|_{t} \leq M\|y\|_{t} \quad \text { for every } y \in F_{t},
$$

where $M=\|g\|_{\infty}$. Thus for each $t \in \mathbb{R}, A_{t}$ is a bounded linear operator on $F_{t}$, and hence $A_{t} \in B\left(F_{t}\right)$. Also, $\left\|A_{t}\right\|_{t} \leq M$ for every $t \in \mathbb{R}$. Let $w$ be the function on $\mathbb{R}$ defined by $w(t)=\|\cdot\|_{t}$. Then $w$ is a weight on $\mathbb{R}$. Consider the set $V=\{\lambda w: \lambda>0\}$. Then $V$ is a system of weights on $\mathbb{R}$. We denote by $L(\mathbb{R})$ a vector space of cross-sections over $\mathbb{R}$. It readily follows that the weighted space $L V_{0}(\mathbb{R})$ is a normed linear space with respect to the system of weights $V$ on $\mathbb{R}$.

Fix $s \in \mathbb{R}$ and define the operator-valued mapping

$$
\psi_{s}: \mathbb{R} \rightarrow \bigcup_{t \in \mathbb{R}}\left(B\left(F_{t}\right)\right)
$$

as

$$
\psi_{s}(t)=e^{s A_{t}} \quad \text { for every } t \in \mathbb{R} .
$$

To prove that each $\psi_{s}$ induces a multiplication operator on $L V_{0}(\mathbb{R})$, in view of Theorem 3.4, it is enough to show that for every $v \in V$ there exists $u \in V$ such that

$$
v_{t}\left(\psi_{s}(t) y\right) \leq u_{t}(y) \quad \text { for every } t \in \mathbb{R} \text { and } y \in F_{t} .
$$

Let $v \in V$. Then $v=\lambda w, \lambda>0$. For $t \in \mathbb{R}$ and $y \in F_{t}$, we have

$$
v_{t}\left(\psi_{s}(t) y\right)=\lambda\left\|e^{s A_{t}}(y)\right\|_{t} \leq \lambda e^{|s| M}\|y\|_{t}=u_{t}(y),
$$

where $u=\lambda e^{|s| M} w$. This proves that $M_{\psi_{s}}$ is a multiplication operator on $L V_{0}(\mathbb{R})$. 
Theorem 5.1. Let $g \in F_{\mathrm{b}}(\mathbb{R})$, and let $\Pi_{g}: \mathbb{R} \times L V_{0}(\mathbb{R}) \rightarrow L(\mathbb{R})$ be the function defined by $\Pi_{g}(s, f)=M_{\psi_{s}} f$ for $s \in \mathbb{R}$ and $f \in L V_{0}(\mathbb{R})$. Then $\Pi_{g}$ is a dynamical system on $L V_{0}(\mathbb{R})$.

Proof. Since for each $s \in \mathbb{R}, M_{\psi_{s}}$ is a multiplication operator on $L V_{0}(\mathbb{R})$, we can conclude that $\Pi_{g}(s, f)$ belongs to $L V_{0}(\mathbb{R})$ whenever $s \in \mathbb{R}$ and $f \in L V_{0}(\mathbb{R})$. Thus $\Pi_{g}$ is a function from $\mathbb{R} \times L V_{0}(\mathbb{R}) \rightarrow L V_{0}(\mathbb{R})$. Also, it readily follows that $\Pi_{g}(0, f)=f$ for every $f \in L V_{0}(\mathbb{R})$, and $\Pi_{g}(s+t, f)=$ $\Pi_{g}\left(s, \Pi_{g}(t, f)\right)$ for every $s, t \in \mathbb{R}$ and $f \in L V_{0}(\mathbb{R})$. To show that $\Pi_{g}$ is a dynamical system, it suffices to prove that $\Pi_{g}$ is continuous. Let $\left(s_{n}, f_{n}\right)$ be a sequence in $\mathbb{R} \times L V_{0}(\mathbb{R})$ such that $\left(s_{n}, f_{n}\right) \rightarrow(s, f)$. Let $v \in V$. Then $v=\lambda w, \lambda>0$.

$$
\begin{aligned}
& \left\|\Pi_{g}\left(s_{n}, f_{n}\right)-\Pi_{g}(s, f)\right\|_{v} \\
& \quad=\sup \left\{\lambda\left\|\psi_{s_{n}}(t) f_{n}(t)-\psi_{s}(t) f_{n}(t)+\psi_{s}(t) f_{n}(t)-\psi_{s}(t) f(t)\right\|_{t}: t \in \mathbb{R}\right\} \\
& \quad \leq \sup \left\{e^{|s| M}\left(e^{\left|s_{n}-s\right| M}-1\right) v_{t}\left[f_{n}(t)\right]: t \in \mathbb{R}\right\} \\
& \quad+\sup \left\{e^{|s| M} v_{t}\left[f_{n}(t)-f(t)\right]: t \in \mathbb{R}\right\} \\
& \quad=e^{|s| M}\left(e^{\left|s_{n}-s\right| M}-1\right)\left\|f_{n}\right\|_{v}+e^{|s| M}\left\|f_{n}-f\right\|_{v} \rightarrow 0
\end{aligned}
$$

as $\left|s_{n}-s\right| \rightarrow 0$ and $\left\|f_{n}-f\right\|_{v} \rightarrow 0$. This proves the continuity of $\Pi_{g}$, and hence $\Pi_{g}$ is a (linear) dynamical system on $L V_{0}(\mathbb{R})$. This completes the proof of the theorem.

Remark 5.2. Consider the family $\left\{M_{\psi_{s}}: s \in \mathbb{R}\right\}$ of the multiplication operators on the weighted space $L V_{0}(\mathbb{R})$ of cross-sections. We observe the following:

(i) $M_{\psi_{s+t}} f=M_{\psi_{s}}\left(M_{\psi_{t}} f\right)$ for every $f \in L V_{0}(\mathbb{R})$ and $s, t \in \mathbb{R}$.

(ii) $M_{\psi_{0}} f=f$ for all $f \in L V_{0}(\mathbb{R})$.

(iii) $\lim _{t \rightarrow 0} M_{\psi_{t}} f=f$ for all $f \in L V_{0}(\mathbb{R})$.

Thus the family $\left\{M_{\psi_{s}}: s \in \mathbb{R}\right\}$ is a $C_{0}$-group of the multiplication operators on $L V_{0}(\mathbb{R})$ which turns out to be locally equicontinuous. To show this, we need to establish that for any fixed $s \in \mathbb{R}$ the subfamily $\left\{M_{\psi_{t}}:-s \leq t \leq s\right\}$ is equicontinuous on $L V_{0}(\mathbb{R})$. Let $s \in \mathbb{R}$ be fixed. Then the family $\left\{M_{\psi_{t}}:-s \leq\right.$ $t \leq s\}$ is a bounded set in $B\left(L V_{0}(\mathbb{R})\right)$ since the mapping $t \rightarrow M_{\psi_{t}}$ is continuous, where $B\left(L V_{0}(\mathbb{R})\right)$ is the locally convex space of all continuous linear operators on $L V_{0}(\mathbb{R})$ with the strong operator topology. Also, for each $f \in L V_{0}(\mathbb{R})$ the set $\left\{M_{\psi_{t}} f:-s \leq t \leq s\right\}$ is bounded in $L V_{0}(\mathbb{R})$, and hence by a corollary of the Banach-Steinhaus Theorem [10, Theorem 2.6] it follows that the family $\left\{M_{\psi_{t}}:-s \leq t \leq s\right\}$ is equicontinuous, and hence the $C_{0}$-group $\left\{M_{\psi_{t}}: t \in \mathbb{R}\right\}$ is locally equicontinuous.

\section{REFERENCES}

1. M. B. Abrahamse, Multiplication operators: Hilbert space operators, Lecture Notes in Math., vol. 693, Springer-Verlag, New York, 1978, pp. 17-36.

2. K. D. Bierstedt, The approximation-theoretic localization of Schwartz's approximation property for weighted locally convex function spaces and some examples, Functional Analysis, Holomorphy and Approximation Theory, Lecture Notes in Math., vol. 843, Springer-Verlag, New York, 1981, pp. 93-149.

3. S. Machado and J. B. Prolla, The general complex case of the Bernstein-Nachbin approximation problem, Ann. Inst. Fourier (Grenoble) 28 (1978), 193-206. 
4. L. Nachbin, S. Machado, and J. B. Prolla, Weighted approximation, vector fibrations and algebras of operators, J. Math. Pures Appl. (9) 50 (1971), 299-323.

5. L. Nachbin, J. B. Prolla, and S. Machado, Concerning weighted approximation, vector fibrations, and algebras of operators, J. Approx. Theory 6 (1972), 80-89.

6. J. B. Prolla, The approximation property for Nachbin spaces, approximation theory and functional analysis, Proc. Conf. Approximation Theory (Campinas 1977), North-Holland Math. Stud., vol. 35, North-Holland, Amsterdam and New York, 1979, pp. 371-382.

7. R. K. Singh and J. S. Manhas, Multiplication operators on weighted spaces of vector-valued continuous functions, J. Austral. Math. Soc. Ser. A 50 (1991), 98-107.

8. __ Multiplication operators and dynamical systems, J. Austral. Math. Soc. Ser. A 53 (1992), 92-102.

9.

10. Walter Rudin, Functional analysis, Tata McGraw-Hill, New York, 1973.

Department of Mathematics, University of JAMmu, Jammu, 180 004, INDia 